\title{
Quantitative atomic-scale profiles of oxygen vacancies in $\mathrm{SrTiO}_{3}$
}

\author{
D. A. Muller,* A. Ohtomo**, J. L. Grazul* and H. Y. Hwang*
}

*Bell Labs, Lucent Technologies, 700 Mountain Ave., Murray Hill, NJ 07974-0636

**Institute for Materials Research, Tohoku University,2-1-1 Katahira, Aoba, Sendai, Japan

Oyxgen vacancies $\left(\mathrm{V}_{\mathrm{O}}\right)$ can act as n-type dopants in $\mathrm{SrTiO}_{3}$, allowing carrier mobilities in excess of $10^{4} \mathrm{~cm}^{2} /(\mathrm{Vs})$ at low temperatures. These dopants must be controlled and confined at the nanometer length-scales in order to produce oxide heterostructures[1]. Using pulsed-laser deposition in ultrahigh vacuum we have been able to produce complex oxide heterostructures with unit-cell precision $[2,3]$. In order to measure the oxygen diffusion that occurred during growth, as well as the efficiency of the self-doping of vacancies, we grew $\mathrm{SrTiO}_{3-\delta}$ films with precisely controlled thickness and known electrical properties. Typical useful vacancy ranges are 1-4\%, which is too low to be detected by the recently proposed "direct" imaging[4] in a Cs-corrected instrument. Instead, we have investigated "indirect" methods with increased sensitivity and sub-nanometer resolution that are more quantitative and relevant to the electrical properties of $\mathrm{SrTiO}_{3}$.

Electron energy loss spectroscopy (EELS) provides information on both the $\mathrm{O}$ and Ti states. Ratios of integrated edge intensities are not practical as the errors are usually a few \%. Instead, fig. 1a shows the $\mathrm{O}-\mathrm{K}$ edge fine structure washes out with increasing $\mathrm{V}_{\mathrm{O}}$ concentration, and even at $\delta=0.13\left(4 \% \mathrm{~V}_{\mathrm{O}}\right)$ changes are visible. Even more useful is the Ti-L edge (fig. 1b). Each oxygen vacancy nominally "transfers" $2 \mathrm{e}^{-}$to the Ti $\mathrm{d}$ band. As there are 3 times as many $\mathrm{O}$ sites as Ti, this is an amplification factor of 6 in fractional detection sensitivity. The Ti valence can be extracted by a MLS fit to $3^{+}$and $4^{+}$reference spectra[3] with a detect limit of $\sim \delta=0.05$ (i.e. about $1 \% \mathrm{~V}_{\mathrm{O}}$ ).

While high-angle annular dark field (HAADF) imaging is sensitive mostly to atomic number (fig. 2a), the low angle dark field (LAADF) signal can detect the strain field from individual vacancies by its distortion of the adjacent cation sites and subsequent dechanneling of the electron beam (fig. $2 b$ ). From the simultaneously recorded bright-field image, the sample in fig. $2 b$ is $10 \pm 2$ $\mathrm{nm}$ thick which means the average number of oxygen vacancies per column is 3 . Fig. $2 \mathrm{c}$ shows a thicker region that averages over more vacancies in the beam direction.

Fig. 3a shows the RHEED pattern used to grow exactly 25 layers of oxygen deficient $\mathrm{SrTiO}_{3-}$ $\delta$. By counting back from the La marker layer in fig. $2 \mathrm{~b}$, the original substrate interface can be located. This is done in fig. $3 \mathrm{~b}$ which shows the average line profile from fig. $2 b$. From a gaussian fit to the interface width, the average diffusional/cluster broadening is at most $0.38 \mathrm{~nm}$ (about 1 unit cell). Fig. 3c shows the electrical width from the Ti EELS signal is $1 \mathrm{~nm}$, consistent with the measured screening length in delta-doped $\mathrm{SrTiO}_{3}[3]$. The SNR in both these line scans give an estimate of the absolute detection sensitivity that is 1-3 and 2-4 oxygen vacancies for LAADF and EELS respectively. In addition, the EELS provides a quantitative measure of charge. Finally, under the appropriate growth conditions, oxygen-doping profiles can be produced with nanometer abruptness, limited only by the fluctuations caused by vacancy clustering.

[1] I. Pallecchi, et al., Appl. Physics Lett. 78, 2244 (2001).

[2] A. Ohtomo, et al., Appl. Physics Lett. 80, 3922 (2002).

[3] A. Ohtomo, et al., Nature 419, 378 (2002).

[4] C. L. Jia, M. Lentzen, and K. Urban, Science 299, 870 (2003). 

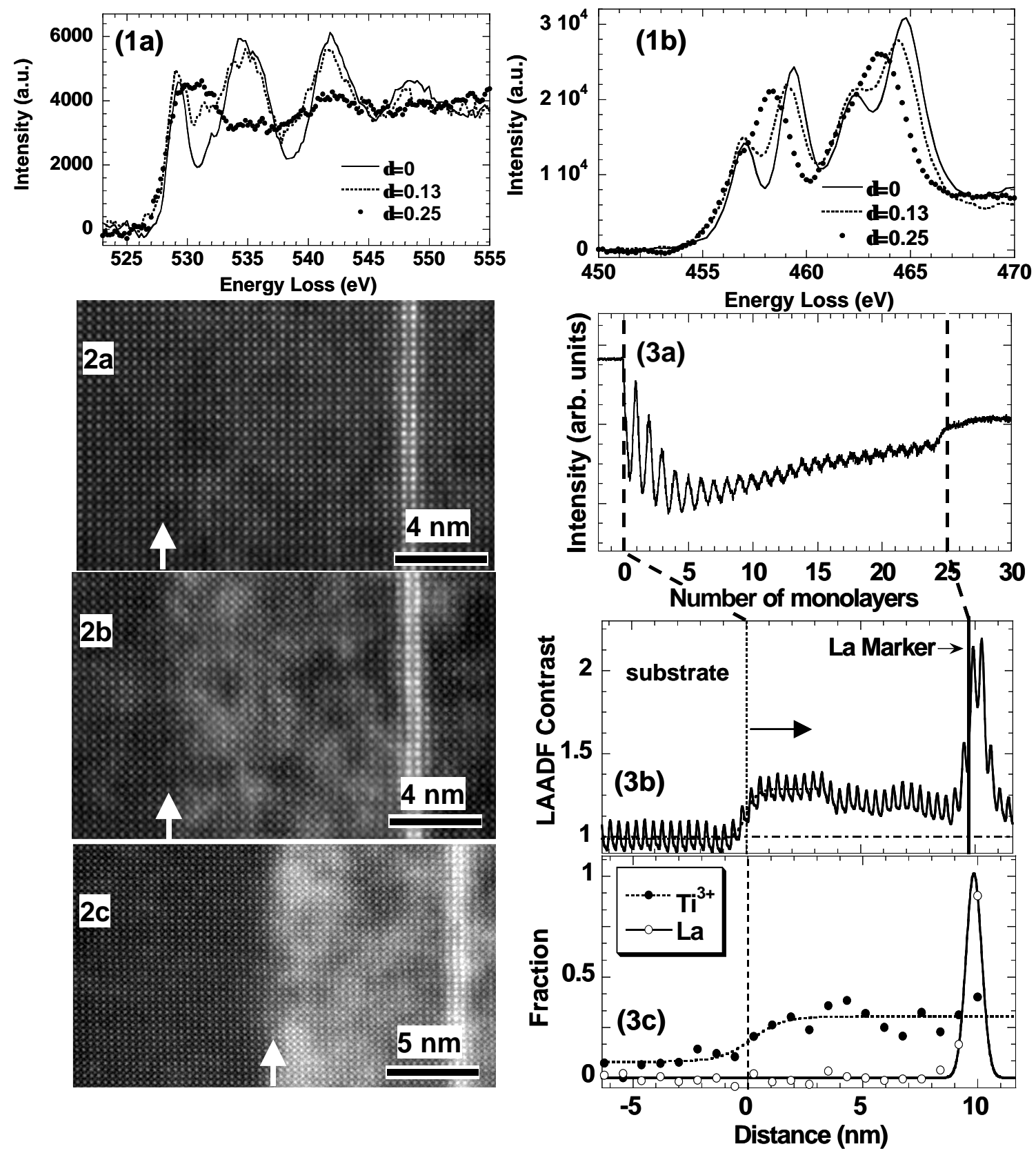

FIG 1. EELS spectra for oxygen deficient $\mathrm{SrTiO}_{3-\delta}$, for $\delta=0,0.13,0.25$. (a) The O-K edge fine structure is sensitive to $\mathrm{O}-\mathrm{O}$ ordering. (b) The Ti-L edge shifts from a $4+$ to $3+$ valence with $\left[\mathrm{V}_{\mathrm{O}}\right]$.

FIG 2. 25 layers of oxygen-deficient $\mathrm{SrTiO}_{3-\delta},(\delta=0.13)$ are grown on bulk $\mathrm{SrTiO}_{3}$ (left of white arrow) and capped with a 2-layer thick $\mathrm{LaTiO}_{3}$ marker layer (right) and more $\mathrm{SrTiO}_{3-\delta}$. (a) HAADF shows only the La marker layer. (b) in LAADF, $\mathrm{V}_{\mathrm{O}}$ clusters in the oxygen-deficient layer are now also visible - this sample is $10 \mathrm{~nm}$ thick. (c) LAADF image in a $\sim 30 \mathrm{~nm}$ thick sample.

FIG 3. Quantitative line profiles though the oxygen-deficient layer. (a) Reflection high-energy electron diffraction (RHEED) pattern during growth shows 25 layers are grown. (b) LAADF line profile -the original interface at $0 \mathrm{~nm}$ is located by counting 25 layers back from the La marker. (c) Ti-L edge EELS profile showing the Ti valence and the simultaneously recorded La M edge. 